\title{
Organ Graft
}

National Cancer Institute

\section{Source}

National Cancer Institute. Organ Graft. NCI Thesaurus. Code C122934.

The transfer of an organ, or organ subpart, from one body to another. 